\title{
Cutaneous tube ureterostomy: a fast and effective method of urinary diversion in emergency situations
}

This article was published in the following Dove Press journal:

Research and Reports in Urology

II June 2015

Number of times this article has been viewed

Tamer Abdin'

Gideon Zamir ${ }^{2}$

Alon Pikarsky ${ }^{2}$

Ran Katz'

Ezekiel H Landau'

Ofer N Gofrit'

'Department of Urology, ${ }^{2}$ Department of General Surgery, Hadassah Hebrew University Hospital, Jerusalem, Israel
Correspondence: Ofer N Gofrit Department of Urology, Hadassah Hebrew University Hospital, PO Box I2000, Ein Kerem, Jerusalem 9| I20, Israel

Email ogofrit@gmail.com
Aim: To report on a simple and rapid method of urinary diversion. This method was applied successfully in different clinical scenarios when primary reconstruction of the ureters was not possible.

Materials and methods: The disconnected ureter is catheterized by a feeding tube. The tube is secured with sutures and brought out to the lateral abdominal wall as cutaneous tube ureterostomy (CTU).

Results: This method was applied in three different clinical scenarios: a 40-year-old man who sustained multiple high-velocity gunshots to the pelvis with combined rectal and bladder trigone injuries and massive bleeding from a comminuted pubic fracture. Damage control included colostomy and bilateral CTUs. A 26-year-old woman had transection of the right lower ureter during abdominal hysterectomy. Diagnosis was delayed for 3 weeks when the patient developed sepsis. The right kidney was diverted with a CTU. A 37-year-old male suffered from bladder perforation and hemorrhagic shock. Emergency cystectomy was done and urinary diversion was accomplished with bilateral CTUs. In all cases, effective drainage of the urinary system was achieved with normalization of kidney function.

Conclusion: When local or systemic conditions preclude definitive repair and damage control surgery is needed, CTU provides fast and effective urinary diversion.

Keywords: Ureter, trauma, tube cutenuous ureterostomy

\section{Introduction}

The ureter is involved in less than $1 \%$ of all urological traumas. ${ }^{1}$ Ureteral injuries are caused by either external trauma (penetrating or blunt) or more commonly by iatrogenic surgical injury. ${ }^{2}$ The optimal management of ureteral injuries is immediate primary surgical repair. However, shock, hypothermia, acidosis, and coagulopathy commonly associated with multiple trauma often does not allow definitive ureteral repair, mandating a modified and abbreviated approach - "damage control" surgery.,

Emergent cystectomy is indicated in rare occasions of bladder necrosis and massive bleeding that cannot be controlled otherwise. ${ }^{5,6}$ Urinary diversion which usually requires the use of bowel segments is very problematic in these situations and usually involves cutaneous ureterostomies. ${ }^{5,6}$ Herein, we present an effective and rapid method for urinary diversion-cutaneous exteriorization ureterostomies using a tube.

\section{Materials and methods}

The technique of cutaneous tube ureterostomy (CTU) can be applied to one or both ureters, with or without removal of the bladder. Trans-peritoneal or retro-peritoneal approach can be used according to the clinical scenario. The transected ureter is catheterized 
with a ureter catheter or a feeding tube. The tube is secured with several interrupted sutures and is brought to the lateral abdominal wall as a tube ureterostomy. It is of paramount importance to educate the nursing team about this unique type of tube and its delicate care. When a patient's condition stabilizes it is possible to insert percutaneous nephrostomy (instillation of contrast medium through the CTU can assist in this procedure). The CTU can either be removed or kept close on the skin. Reconstructive surgery can be planned after the patient has recovered from the acute condition. This surgery was done in three different clinical scenarios.

\section{Results}

The method of CTU was used in three cases treated in our institution.

Case 1: a 26-year-old previously healthy woman, a mother of seven children, was transferred from another hospital in a state of septic shock. Three weeks earlier, the patient developed massive postpartum hemorrhage following spontaneous vaginal delivery and emergent total abdominal hysterectomy was done. Postoperatively, the patient developed severe lower abdominal and right flank pain, chills, and vomiting and was transferred to our institution. Physical examination disclosed a hemodynamically stable patient with temperature of $38.90^{\circ} \mathrm{C}$ and a lower abdominal tender mass which was palpable also on vaginal examination. Blood tests revealed white blood count of 23,000 per $\mathrm{mm}^{3}$ and hemoglobin level of $10.6 \mathrm{~g} / \mathrm{dL}$. Blood biochemistry including renal function tests were within normal limits. The patient was taken to the operating room by a gynecological team with a presumed diagnosis of Douglas pouch abscess, but puncture of the posterior fornix yielded urine. Urologic consultation was requested. Cystoscopy revealed normal urethra, bladder, and ureteral orifices. Retrograde urethrography showed normal left ureter and collecting system and complete transection of the right ureter $5 \mathrm{~cm}$ above the bladder level. Cystogram was normal. Midline laparotomy revealed a large urinoma in the midline (Figure 1A). The small bowel was edematous

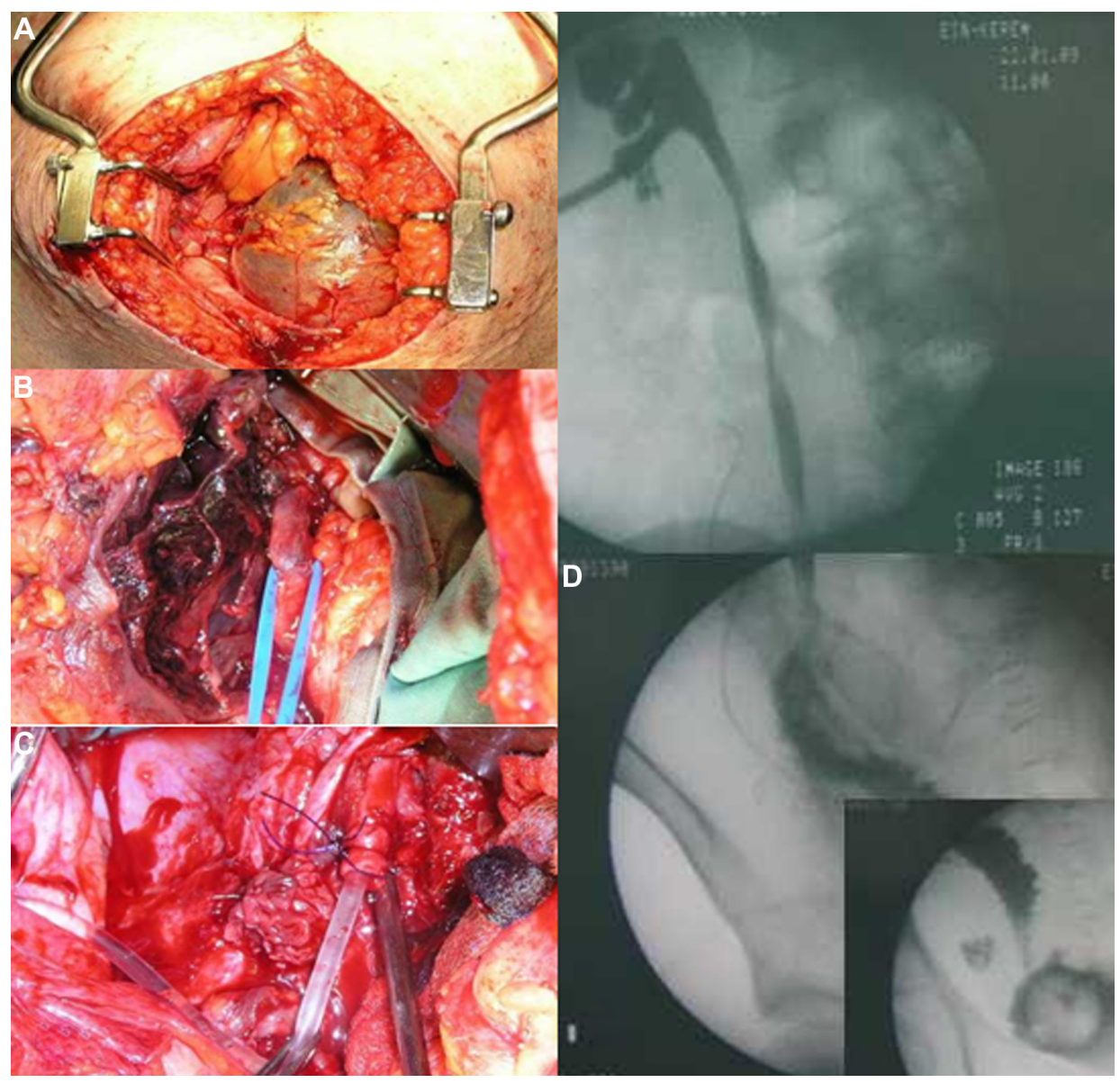

Figure I Photos of Case I.

Notes: (A) Intra-operative view showing a large midline urinoma. (B) Isolation of the ureter above the hematoma. (C) Intubation of the ureter with a I0 Fr feeding tube. (D) Nephrostogram done after the definitive surgery demonstrating passage of contrast from the kidney to the ureter and then to the bladder through an ileal segment (a mosaic of three fluoroscopy fields). 
and covered with fibrin. In addition, several large hematomas surrounding the right ureter, which was draining urine freely, were noticed. Due to the septic condition of the patient and the presence of severely inflamed small bowel, it was decided not to perform a definitive repair. The ureter was dissected higher until a normal ureter was found (Figure 1B). A $10 \mathrm{Fr}$ feeding tube was placed in the right ureter and secured with polydioxanone $2 / 0$ sutures (Figure $1 \mathrm{C}$ ). The distal end of the tube was brought out to the lateral abdominal wall as CTU. The postoperative course was uneventful. White blood count normalized the next day and the patient resumed normal diet on the second postoperative day. On the eighth postoperative day, a right nephrostomy tube was placed, the tube ureterostomy was closed on the skin and the patient discharged home. Three months later an ileal interposition was performed uneventfully (Figure 1D).

Case 2: a 40-year-old male sustained multiple gunshot wounds during a terrorist attack. Several entrance wounds were noticed in the buttock and a single exit wound above the pubic symphysis was seen. Upon admission, the patient was neurologically intact, but tachycardic. Rectal examination showed bloody discharge. After initial fluid resuscitation the patient was transferred to the operating room and a laparotomy performed. Combined rectal and urological injuries were evident including wide, bleeding tears of the rectum and bladder trigone. A comminuted pubic fracture was also evident. Shortly after opening the peritoneal cavity, the patient bled profusely from the pelvis and developed hemorrhagic shock despite massive transfusion. A "damage control" procedure was carried out. The rectum was closed with a stapler and end sigmoid diversion colostomy was constructed. The bladder was explored; the left ureteral orifice was identified and catheterized while the right one could not be identified. The bladder was closed rapidly with a single suture line. The ureters were identified and transected at the level of the common iliac vessels and brought out to the abdominal wall as tube ureterostomies. Temporary abdominal closure using a plastic bag was carried out. The postoperative course was remarkable for severe sepsis with multi-organ failure. Repeated open debridement of pelvic necrotic tissue and abscess drainage was performed. During this period urinary output through the ureterostomies was satisfactory with normal renal function. Three weeks after the injury the patient's condition stabilized. The tube ureterostomies were removed after the insertion of nephrostomies. Later, the abdomen was closed with skin graft and the patient discharged home with two opened nephrostomies and a well-functioning colostomy.
One year after the injury, evaluation revealed vesicorectal fistula, small bladder capacity $(100 \mathrm{cc})$ with left vesicoureteral reflux to the point of the transection, normal anal and urethral sphincters and normal erection. Definitive surgery included division of the fistula, closure of colostomy, and anastomosis of the proximal colon to the lower rectum, left uretero-ureterostomy, augmentation ileocystoplasty, right ileal interposition, and protecting ileostomy. Postoperative course was unremarkable, and a year later the ileostomy was closed. Four years later, the patient is potent and fully continent.

Case 3: a 37-year-old male with multiple sclerosis treated with high-dose steroids was admitted through the emergency department with urosepsis, urinary retention, and acute renal failure. The patient was hospitalized in the intensive care unit and a catheter was inserted. Two days after admission, the patient developed peritonitis and hemorrhagic shock. Computed tomography (CT) showed perforation of the bladder. Blood products and fluids were given and emergent laparotomy was done. Intra-peritoneal bladder tear was found and repaired in multiple layers. After surgery, bleeding continued and a cystogram showed a massive leak from the suture line. A second attempt to close the bladder was done 2 days after the first surgery. The patient's condition continued to be critical with blood product consumption and renal failure with creatinine level that reached $915 \mathrm{mmol} / \mathrm{L}$. Another cystogram showed that the bladder was completely open. A third laparotomy was done 2 days after the second surgery. Massive bleeding from multiple points in the bladder was noticed. The ureteral orifices were catheterized with feeding tubes number 8 and cystectomy was done. The ureteral catheters were brought out of the abdominal wall as CTUs. The patient's hemodynamic condition stabilized, blood consumption ceased, and a high urinary output through the ureterostomies was recorded with a decrease in creatinine level to $133 \mathrm{mmol} / \mathrm{L}$. On the third day after the third surgery focal face convulsion was noticed. CT showed diffuse brain edema. This was attributed to sepsis. The patient's general condition deteriorated and he died 7 days after the third operation. Pathological examination of the bladder showed atrophic detrusor muscle and an absence of the mucosal lining.

\section{Discussion}

Emergent diversion of the urine may be required in diverse clinical scenarios. These include traumatic ureteral injuries (external or iatrogenic) and non-traumatic instances. In all cases the patient is critically ill and it is impossible to perform 
complex reconstructive surgery. These occurrences, however, are rare, and the personal experience of most urologists in their management is usually limited.

The concept of "damage control surgery" evolved in the early 1990s. It implies doing only the absolutely essential to control exsanguination and to prevent spillage of the urine and intestinal contents into peritoneal cavity. Definitive repair and complex procedures are postponed. ${ }^{7}$ A key factor for the success of this strategy is the early recognition that the patient cannot tolerate a definitive procedure. "Damage control" strategy should be applied to patients with hemodynamic instability and multiple visceral injuries. ${ }^{8}$ In urology, damage control usually implies the efficient control of urinary extravasation. ${ }^{9}$ This can be accomplished by several means:

a) Ignoring the ureteral injury, and planning for re-operation when the patient's condition stabilizes.

b) Ligating the proximal ureter and placing a nephrostomy tube, either intra-operatively or postoperatively. This decision is risky and may result in renal failure (in bilateral cases), or kidney loss (if unilateral). The intra-operative placement of nephrostomy tube is technically difficult, and later transfer from the intensive care unit to the angiography suit may not be possible.

c) Placing an internal ureteral stent, when feasible.

d) Exteriorizing the ureter directly to the skin. ${ }^{5,6}$ This procedure is not advised since it is lengthy; it requires long dissection of the proximal ureter with possible compromise of its vascularization and loss of ureteral length which may interfere with definite reconstructive surgery.

e) Exteriorizing the ureter to the skin over a tube as done in the cases presented here.

Using a feeding tube as tube ureterostomy was suggested by Gill and McRoberts, ${ }^{9}$ and using a single J stent by Coburn. ${ }^{10}$ Detailed description of this procedure is not reported in the literature. CTU is a rapid procedure. Renal failure or renal loss due to obstructive uropathy is avoided and the distal ureter is left unharmed for possible future reconstructive surgery.

In the cases presented here, damage control surgery with CTUs was done in three completely different clinical situations (penetrating trauma, iatrogenic trauma, and emergent cystectomy). In all instances, effective urinary diversion was accomplished with normalization of kidney function that greatly promoted patients' recovery. Regrettably, the last patient expired due to intracranial complication of the septic process.

CTUs should be managed with great care and the nursing staff must be aware of their delicacy. Conversion to nephrostomies should be made as soon as patient's condition stabilizes. The decision whether to leave the tube nephrostomy dwelling or to remove it is dependent on the patient's condition. When reconstructive surgery can be planned soon (as in the first case), the CTU is better kept dwelling (closed on the skin) for facilitating ureteral identification during the reconstructive surgery. However, if reconstructive surgery cannot be planned (as in the second and third cases) it is better to remove the CTU after nephrostomy insertion.

\section{Conclusion}

When local or systemic conditions preclude definitive repair, and damage control surgery is needed, CTU provides fast and effective urinary diversion. Minimal damage is caused to the ureter which can be reconstructed when the patient recovers.

\section{Disclosure}

All the authors declare that there is no potential conflict of interests or any financial relation with the commercial identities mentioned in the paper.

\section{References}

1. Presti JC Jr, Carroll PR, McAninch JW. Ureteral and renal pelvic injuries from external trauma: diagnosis and management. $J$ Trauma. 1989;29(3):370-374.

2. Lezin MA, Stoller ML. Surgical ureteral injuries. Urology. 1991;38(6): 497-506.

3. Medina D, Lavery R, Ross SE, Livingston DH. Ureteral trauma: preoperative studies neither predict injury nor prevent missed injuries. J Am Coll Surg. 1998;186(6):641-645.

4. Franko ER, Ivatury RR, Schwalb DM. Combined penetrating rectal and genitourinary injuries: A challenge in management. J Trauma. 1993; 34(3):347-353.

5. Fujiwara S, Noguchi T, Noguchi T, Emoto A, Tasaki Y. Panperitonitis caused by gangrene of the urinary bladder: report of a successfully treated case. Am Surg. 2008;74(4):302-304.

6. Bonera A, Antoci G, Bianchi P, Alberti P. [Gangrenous cystitis. Case report]. Minerva Urol Nefrol. 1996;48(4):193-197. Italian.

7. Hirshberg A, Mattox KL. Damage control in trauma surgery. Br J Surg. 1993;80(12):1501-1502.

8. Rotondo MF, Zonies DH. The damage control sequence and underlying logic. Surg Clin North Am. 1997;77(4):761-777.

9. Gill IS, McRoberts JW. New directions in the management of GU trauma. Mediguide Urol. 1992;5:1-8.

10. Coburn M. Damage control for urologic injuries. Surg Clin North Am. 1997;77(4):821-834. 
Research and Reports in Urology

\section{Publish your work in this journal}

Research and Reports in Urology is an international, peer-reviewed, open access journal publishing original research, reports, editorials, reviews and commentaries on all aspects of adult and pediatric urology in the clinic and laboratory including the following topics: Pathology, pathophysiology of urological disease; Investigation and treatment of

urological disease; Pharmacology of drugs used for the treatment of urological disease. The manuscript management system is completely online and includes a very quick and fair peer-review system, which is all easy to use. Visit http://www.dovepress.com/testimonials.php to read real quotes from published authors.

Submit your manuscript here: http://www.dovepress.com/research-and-reports-in-urology-journal 